\title{
INFORMES
}




\section{ALGUNAS REFLEXIONES SOBRE LA APLICACION DE LOS COMPUTADORES EN LA EDUCACION*}

Francisco Rueda F.**

\section{Introducción}

Leía recientemente la opinión de un respetable humanista que invitaba a sus colegas a preocuparse de la técnica, argumentando que la actitud opuesta (ignorarla, o negarla, o limitarse a atacarla) puede tener efectos bastante negativos para la sociedad. Creo que no hay que forzar mucho esta idea para concluir que es importante que los pedagogos nos preocupemos de las nuevas tecnologías educativas, precisamente para que no seamos "víctimas" de ellas. Las ideas expuestas en este artículo están inscritas dentro de esta línea de pensamiento.

En los últimos tiempos se ha venido hablando mucho de la aplicación de los computadores en la educación, y se han celebrado frecuentemente seminarios y creado varios grupos de trabajo sobre el tema. Sin embargo, en mi opinión, no hay todavía suficiente claridad sobre el asunto, en parte por la complejidad inherente al mismo y en parte por la invasión de información que hemos recibido sin que hayamos tenido oportunidad de digerirla completamente. El presente artículo tiene por objetivo hacer algunos planteamientos sobre el asunto, con el ánimo de buscar una mayor claridad al respecto. Las experiencias presentadas son el fruto de un proceso relativamente corto de reflexión y trabajo sobre el tema, que ha sido desarrollado por un grupo de profesores de la Universidad de Los Andes desde hace algunos meses y que nos ha servido para aclarar las ideas y para ir definiendo con mayor nitidez nuestra posición al respecto, y nuestros planes y proyectos futuros.

Una primera distinción que me parece conveniente hacer es entre la Informática como soporte a la docencia (lo que tradicionalmente se ha dado en llamar CAI o CAL o EAG) y la Informática como objeto de estudio. En el primer caso, se busca identificar aquellos aspectos en los que la tecnología computacional puede dar una respuesta adecuada a los problemas docentes, y el segundo surge al constatar la enorme importancia de la Informática y la imperiosa necesidad que tenemos de hacerla accesible al común de la gente, dada su ubicuidad y su generalizada aplicación a todos los sectores de la sociedad. El presente artículo se referirá al primer aspecto.

Una de las moralejas del artículo es que los computadores tienen un campo de acción prácticamente ilimitado en la educación, pero que es importante conocer los diferentes caminos posibles, con sus muchas sinuosidades, restricciones y destinos posibles, para que los frutos recogidos puedan ser los adecuados. Dentro de esta perspectiva calificar de buena o mala la aplicación de los computadores a la educación es una afirmación tan general que carece de significado real, o decir que la solución a todos los problemas de Informática Educativa puede encontrarse en un determinado lenguaje, afirmación que a veces hacen los menos informados en el asunto, refleja una subestimación de las posibilidades de la tecnología computacional para enfrentar problemas educativos.

Presentamos a continuación un intento de definición de los enfoques presentados al problema; un recuento de algunas teorías educativas que pueden servir como base para

\footnotetext{
* Ponencia presentada en el Seminario "Para democratizar la informática", organizado por el SENA, marzo, 1984.

** Departamento de Ingeniería de Sistemas, Universidad de Los Andes.
} 
los desarrollos de la Informática Educativa; una descripción de algunos aspectos que deben tenerse en cuenta al construir sistemas computarizados de ayuda a la docencia, y, finalmente, algunas conclusiones generales.

\section{Los enfoques de la informática educativa}

Los desarrollos en el área de Informática Educativa podemos clasificarlos en dos grandes grupos, cuya diferenciación, a pesar de no ser muy neta en algunos casos, introduce, en mi opinión, una mayor claridad en el asunto.

Por un lado, esta el enfoque que podríamos llamar descendente (tomando prestada una terminología típicamente informática) el cual, partiendo de las concepciones más generales -en el caso de un curso, por ejemplo, cuáles son sus objetivos, cuál es la mejor forma de presentar los diferentes conceptos, qué se espera de los estudiantes, cuál es el contexto en que se va a desarrollar, etc.- utiliza el computador como un medio más, y trata de identificar aquellos aspectos en que puede ser superior a los otros en el proceso del aprendizaje.

Al realizar el proceso anterior, se encuentra muy a menudo, como resultado paralelo, que de todos los medios disponibles (audiovisuales, materiales para autoaprendizaje...) muy pocos son usados en la enseñanza por el docente tradicional.

Este método representa una forma muy gradual de enfocar el problema pero no tiene un poder muy grande de cuestionamiento, por lo que se corre el peligro de construir sistemas que en el mejor de los casos refuerzan los métodos tradicionales, sin aportar ideas novedosas. Además, hay que tener mucho cuidado para no empantanarse en problemas educativos, de gran generalidad y con pocas posibilidades de conducir a implementaciones reales. Un ejemplo típico de proyecto que podría desarrollarse con esta óptica sería el análisis de un curso específico (por ejemplo matemáticas o geometría en un año específico de la primaria), y la identificación de aquellos aspectos en los que el computador puede ser útil.

El enfoque que podemos llamar ascendente consiste en construir herramientas muy generales que aprovechen las múltiples posibilidades de la tecnología computacional para generar ambientes que faciliten el proceso de aprendizaje. Este enfoque conduce usualmente a cuestionar más profundamente los esquemas tradicionales y permite evitar algunos problemas teóricos, aunque presupone de todas maneras toda una filosofía de la educación. El ejemplo más representativo de este punto de vista es LOGO.

En nuestra opinión los dos enfoques mencionados son válidos y útiles, y pueden conducir además a resultados muy positivos, pero es importante conocer sus limitaciones y áreas de aplicación antes de iniciar cualquier desarrollo al respecto.

En el caso del grupo de trabajo al cual pertenecemos se escogió inicial-mente como base para el desarrollo de proyectos el primer esquema, pues nos pareció una vía más fácil para ir familiarizándose con los problemas educativos, antes de emprender proyectos más ambiciosos.

\section{Algunas concepciones teóricas sobre la educación}

Uno de los aspectos más ignorados en las presentaciones que se hacen sobre Computadores y Educación es el de las teorías educativas que sirven como soporte a los 
diferentes métodos. No es nuestra intención hacer una presentación exhaustiva y profunda del tema, pues esto no se podría hacer en un espacio tan limitado, y además nuestra formación no nos permite hacerlo con el rigor que sería necesario, pero sí es importante resaltar que cualquier proyecto de Informática Educativa presupone una filosofía de base que no debe olvidarse en ningún momento del desarrollo o evaluación de un sistema educativo. En esta sección presentaremos algunas ideas al respecto con el propósito de ilustrar el tipo de preocupaciones que deben servir como guía a los constructores y evaluadores de este tipo de sistemas.

Entre las diferentes teorías o vertientes de pensamiento existentes sobre educación, hay dos que han recibido especial atención: el conductismo y el estructuralismo. Veamos cuáles son algunos de los principios básicos de cada una de ellas.

El conductismo, cuyo principal representante es Skinner, considera que el aprendizaje se puede asimilar a un cambio de comportamiento, el cual se puede inducir por medio de técnicas de condicionamiento, entendiendo éste como el "crear y reforzar por el ejercicio y los resultados de la acción un cierto número de asociaciones motrices o verbales juzgadas constitutivas de los conocimientos posteriores"118. No entraremos aquí a discutir la validez de esta afirmación, pero sí enunciaremos algunos principios de aprendizaje que se pueden deducir de ella (en nuestra opinión de gran utilidad en el diseño de sistemas de enseñanza computarizados):

- Es importante definir claramente los objetivos que se espera obtener en el proceso de aprendizaje (o el comportamiento que se va a establecer, según Skinner). Además, como algunos de ellos pueden ser muy complejos, es importante definir objetivos parciales. Esto conduce al concepto de instrucción programada.

- En opinión de Skinner, el proceso de aprendizaje requiere suministrar a quien aprende una gran cantidad de refuerzos muy variados lo cual es imposible de realizar en una sesión de clase tradicional, en parte por el tipo de refuerzos requeridos y en parte porque el docente no puede atender a cada estudiante individualmente. Quien haya participado de alguna forma en el proceso de aprendizaje habrá constatado la veracidad de esta afirmación (por ejemplo, la necesidad que tiene el profesor de "nivelar por lo alto", o "nivelar por el promedio"). Para dar una respuesta a este problema Skinner diseñó la "máquina de enseñar", que funcionaba de la siguiente forma: al estudiante se le mostraba, por ejemplo, una ecuación para completar, y se le daba un teclado con el cual colocaba la respuesta, y una palanca que podía accionar; si la respuesta era correcta, accionaba la palanca y esta le presentaba el siguiente problema; en caso contrario, la palanca no se movía y el estudiante debía ensayar nuevamente con la respuesta. Muchos de los primeros sistemas educativos computarizados funcionaban según un esquema similar (hay todavía sistemas que funcionan de esta forma aunque hoy en día no vacilaríamos en calificarlos de "prehistóricos").

-- Aunque es muy discutible el papel que juegan los refuerzos en el proceso de aprendizaje, es innegable que ellos constituyen un ingrediente más que no puede ser ignorado. También aquí podríamos remitirnos al "salón de clase" para mostrar que tanto un refuerzo positivo (una buena nota) como uno negativo (una contestación "dura" del profesor a una pregunta del estudiante) pueden tener una gran incidencia en el proceso de aprendizaje. Con respecto a esto, Skinner considera que el refuerzo

\footnotetext{
${ }^{118}$ PIAGET J., Ou va l'education, Bibliothèque Médiations, Donoël Gonthier, 1972.
} 
debe darse después de que el estudiante haya dado una respuesta adecuada y no antes. Según él, "una página interesante impresa en cuatro colores refuerza sólo la acción con que el alumno abre el libro y lo mira, pero no la de leer el texto de la página ni tampoco la de examinarla despacio"119. Podemos seguramente sacar algunas enseñanzas de este principio, o del que establece que el refuerzo debe ser "oportuno".

- Un aporte importante de Skinner fue el de "crear una auténtica tecnología educacional" 120 (aunque si somos puristas tenemos que reconocer que la tecnología educacional existe desde hace mucho tiempo si consideramos que el libro fue una de las primeras máquinas de aprender", de donde podemos deducir fácilmente la enorme importancia que tuvo la invención de la imprenta).

En opinión de Piaget, "desde el punto de vista pedagógico, la enseñanza programada conduce a aprender pero no a inventar... Otro tanto se puede decir de los métodos audiovisuales.., los cuales pueden favorecer las asociaciones sin dar lugar a actividades auténticas... Una verdad que se reproduce no es más que una verdad a medias"121.

Otra escuela de pensamiento de gran trascendencia en la educación (y en la Informática Educativa) es el estructuralismo, representado principalmente por Piaget. Sería imposible hacer aquí una exposición completa de sus teorías, pero podemos intentar hacer un recuento de algunos de sus principios educativos:

— Piaget es "constructivista" en el sentido de que considera que el niño va construyendo sus propias estructuras que le permiten ir comprendiendo la realidad en forma cada vez más precisa. Esto implica "el uso de métodos activos que conceden una parte esencial a la investigación espontánea del niño o del adolescente y que exigen que toda verdad que sea adquirida sea reinventada por el estudiante o, por lo cienos, reconstruida y no simplemente transmitida". De ahí el famoso aforismo "comprender es inventar o reconstruir por reinvención"122.

Los principios anteriores se aplican a la educación moral, pues, según Piaget, "si el niño es pasivo intelectualmente no podrá ser libre moralmente" ${ }^{123}$.

Piaget realizó investigaciones especialmente en el área de Matemáticas y Física. Encontró, por ejemplo, que a la edad de 7-8 años el niño puede entender los conceptos de unión, intersección, producto cartesiano y conservación de la cantidad, a los 11-12 los de conjunto de partes de un conjunto y conservación del volumen, etc., pues a esas edades ya ha construido las estructuras adecuadas. El corolario debe ser entonces que no se puede pretender que el niño entienda conceptos que presupongan la existencia de las estructuras anteriores si éstas no han sido adquiridas, y que por lo tanto es conveniente enfrentarlo a situaciones en que pueda construir estas estructuras.

- De acuerdo con los principios anteriores, es fundamental que la educación sea un proceso en que el niño participe activamente para que pueda "inventar o reconstruir por invención" los diferentes conceptos. Es importante señalar que para esto no basta con la experimentación, sino que es necesario que ésta se realice en un ambiente adecuado, el cual debe ser cuidadosamente diseñado de acuerdo con principios pedagógicos y sicológicos.

\footnotetext{
${ }^{119}$ SKINNER B. F., Tecnología de La Enseñanza, Nueva colección Labor, 1970.

${ }^{120}$ Ibídem.

${ }^{121}$ PIAGET J.. Ou va l'education, Bibliothéque Médiations, Donoël Gonthier, 1972.

122 Ibídem.

${ }^{123}$ Ibídem.

Digitalizado por RED ACADEMICA
} 
Una inquietud que puede surgirnos con los principios anteriores es si los maestros van a desaparecer como consecuencia de las actividades autónomas del estudiante. Oigamos la respuesta de Piaget: "el educador es indispensable como animador para crear las situaciones y construir los dispositivos de partida susceptibles de plantear problemas útiles al niño y después para organizar contraejemplos que fuercen a la reflexión y obliguen al control de las soluciones muy apresuradas" ${ }^{124}$.

Una buena pregunta que podemos hacernos es si las teorías "piagetianas" pueden aplicarse universalmente. Cómo hacer, por ejemplo, para aplicarlas en la enseñanza de un idioma o de la Literatura, o de la Historia, dado que en esos casos, evidentemente, no podemos inventarnos" nada. El secreto está en poder identificar cuáles son las estructuras subyacentes que nos permiten aprender esas disciplinas. Veamos un ejemplo al respecto:

La Universidad de Los Andes, conjuntamente con el Consejo Británico, ha diseñado un método para el aprendizaje del inglés escrito que nos hace recordar las "estructuras" de Piaget. A través de diferentes ilustraciones se le presentan al estudiante varios conceptos como los de clase-miembro, todo-partes, términos equivalentes, propiedades cuantificables, propiedades generales, localización, etc., los cuales le permiten, supuestamente, adquirir un mejor conocimiento de textos escritos en inglés. Aunque la información con que contamos no nos permite afirmar a priori que el curso haya sido diseñado de acuerdo con los principios básicos del Estructuralismo, ni que esta sea la mejor forma de enseñar idiomas, sí podemos afirmar que este enfoque está más cercano a esta escuela de pensamiento que, por ejemplo, los tan conocidos métodos audiovisuales.

- Hay dos principios que en opinión de Piaget deben ser tenidos en cuenta en el aprendizaje: el primero, consiste en no resolver problemas en abstracto sino en relación con alguna situación real (en muchos casos estudiantes que no pueden resolver problemas teóricos específicos pueden hacerlo si se les presentan de forma más "natural", lo cual está muy relacionado con la "metamafobia" de que nos habla Papert), y el segundo en no pasar muy rápidamente de los aspectos cualitativos a los cuantitativos (por ejemplo de los principios fundamentalmente de la Física a las ecuaciones que los cuantifican).

Uno de los investigadores que más ha trabajado en el área de Informática Educativa es Papert, autor de LOGO, quien fue discípulo de Piaget, y quien ha venido desarrollando desde hace algunos años investigaciones sobre la aplicación de los computadores en la educación. Algunos de sus principios más importantes son los siguientes:

- "Cualquier cosa es fácil de aprender si uno puede asimilarla a la propia colección de modelos... Lo que un individuo puede aprender, y cómo lo aprende depende de los modelos con que cuenta"125 - Esto no es más que una forma de expresar la teoría "constructivista" de Piaget. Es importante entonces que el individuo cuente con ambientes adecuados que le permitan "construir su propia colección de modelos", y que participe activamente en este proceso.

-En opinión de Papert, una de las mejores formas de aprender es a través del ambiente circundante. Desafortunadamente éste no es siempre el adecuado para favorecer el

\footnotetext{
${ }^{124}$ PIAGET J., Ou va l'education, Bibliothèque Médiations, Donoël Gonthier, 1972.

${ }^{125}$ PAPERT S., Desafio a la mente, Ed. Galápago, 1980.
} 
aprendizaje, por lo que hay que crear ambientes artificiales o "microcosmos". Una muy buena forma de ilustrar esto es considerar el aprendizaje de idiomas extranjeros. Todos sabemos que ningún método de aprendizaje tradicional puede suplantar la experiencia de vivir en el país en donde se habla el idioma (!qué mejor forma hay de aprender el francés que vivir en París!). Este principio puede ser de gran utilidad en el diseño de sistemas de aprendizaje computarizados.

-Uno de los factores que más incide en el aprendizaje es el factor afectivo. Todos sabemos la gran motivación que el computador puede generar en los niños, especialmente cuando se les deja participar, construir, inventar.

-En el mundo de la programación hay una serie de conceptos y técnicas que pueden ser muy instructivas si se aplican al aprendizaje: la depuración, o la acción de construir modelos que pueden irse corrigiendo hasta llegar a ser completamente correctos, sin que esto represente una labor "penosa", el concepto de procedimiento, etc.

-Papert diseñó el lenguaje de programación LOGO basado en sus experiencias de investigación y en las ideas pedagógicas que pudo inducir de ellas. Si miramos superficialmente el asunto podemos afirmar que Logo no es más que una máquina de aprender más. Sin embargo no se puede ignorar el hecho que detrás de él hay toda una filosofía de la educación, lo cual podría ilustrarse con la siguiente afirmación de Papert: "...no debe interpretarse el papel central de la tortuga en este libro en el sentido de que la propongo como panacea para todos los problemas educacionales. La considero un objeto educacional valioso, pero aquí su función principal consiste en servir como modelo para otros objetos aún no inventados ${ }^{126}$.

Una observación final con respecto a los principios pedagógicos de Papert, tomada de uno de sus escritos: "En la medida en que nos es permitido observarlo en estos episodios, el contacto con el computador no ha modificado el modo de pensar en que estas personas reflexionan sobre sí mismas o abordan los problemas. En este libro, analizo las formas en que la presencia de la computadora podría contribuir a los procesos mentales no sólo instrumentalmente, sino de manera más esencial y conceptual, influyendo sobre el modo en que las personas piensan aun cuando se encuentren muy alejadas del contacto físico con el computador"127. En mi opinión este tipo de opiniones, que sirven para ilustrar lo ambicioso de los proyectos de Papert, habría que pasarlas por el tamiz de un proceso de reflexión y experimentación adecuados en nuestro medio, antes de aceptarlas como definitivas.

Para terminar este resumen de algunos principios pedagógicos que pueden servir como base para diseñar sistemas de Educación Asistida por Computador, podemos transcribir una observación de Piaget que creemos suscribirían sin dificultad Skinner y Papert:

“...nuestra hipótesis es que las así llamadas aptitudes que diferencian a los "buenos estudiantes" en Matemáticas o Física, suponiendo un nivel igual de inteligencia, consisten sobre todo en poderse adaptar al tipo de enseñanza que se les da, y que los "malos estudiantes" en estas disciplinas pero que tienen éxito en otras, son completamente aptos para dominar los temas que parecen no entender, pero con la condición de llegar a ellos

\footnotetext{
${ }^{126}$ PAPERT S., Desafio a la mente, Ed. Galápago, 1980.

${ }^{127}$ Ibídem.
} 
por vías distintas, porque, lo que ellos no entienden son las "lecciones" dadas y no la materia"128

\section{Los computadores en la educación}

\subsection{Por qué los computadores son una buena herramienta de soporte a la educación}

De acuerdo con los principios anteriores podemos enunciar algunas de las razones por las cuales los computadores constituyen una muy buena herramienta para el aprendizaje:

- Existe una tendencia de crecimiento en la demanda en las instituciones educativas la cual hace prever que en el futuro no se contará con los suficientes recursos para poderles impartir a todos los estudiantes una educación adecuada. En algunos países como Estados Unidos esto está acompañado por una disminución del número de profesores (según cifras que se han calculado en 1995 habrá un 25\%-30\% menos de profesores que en 1979) ${ }^{129}$. Teniendo en cuenta esto, el computador surge como una tecnología más que puede permitir que la educación se masifique sin que esto tenga preocupantes repercusiones en la calidad de la instrucción impartida.

-El computador tiene una característica que lo hace un medio muy poderoso en el proceso educativo: su posibilidad de interacción con el estudiante. Es este uno de los aspectos que lo ponen en ventaja con respecto a otros medios como el libro, los audiovisuales, la televisión, etc. Más aún, podríamos decir que esta característica le permite hacer cosas que ni el mismo profesor puede hacer en el contexto de la sesión de clase tradicional, como interactuar individualmente con cada uno de los estudiantes. Además, como se vio en la sección anterior, este proceso reviste un interés pedagógico de primer orden. Es por medio de él que se pueden aplicar principios educativos como el famoso "variar el tiempo de aprendizaje pero mantener el nivel de éste constante" (por oposición al sistema tradicional de "mantener constante el tiempo y variar el nivel de aprendizaje, dependiendo de las capacidades de cada estudiante"), el cual nos permite eliminar el "darwinismo" académico de que nos habla Skinner ${ }^{130}$. No hay que descartar, sin embargo, la posibilidad de utilizar sistemas que combinen diferentes métodos de trabajo (por ejemplo el profesor puede supervisar el trabajo de cada uno de sus alumnos con un sistema de aprendizaje soportado por un computador).

-El computador posee características que le dan grandes potencialidades: posibilidad de efectuar cálculos en forma veloz, de almacenar gran cantidad de información, de realizar y almacenar gráficas y de realizar "animación", de presentar diferentes secuencias del material de acuerdo con la elección del estudiante, de comunicarse con sitios remotos en forma instantánea. Si analizamos los diferentes medios veremos que ninguno tiene todas esas propiedades (¡ni aún el profesor!).

- Ya empieza a vislumbrarse la confluencia de varias tecnologías: la computacional, la de telecomunicaciones, la producción de cine, la publicidad, etc. lo cual, sobra decir, amplificará en forma imprevisible las potencialidades de todas ellas.

\footnotetext{
${ }^{128}$ PIAGET J., Ou va l'education, Bibliothèque Médiations, Donoël Gonthier, 1972.

${ }^{129}$ O’Neil Jr. Computer Based Instruction, A state-of-the-art assessment, Editado por HF. O’Neil Jr., Ed Academic Press, 1981.

${ }^{130}$ SKINNER B. F., Tecnología de la enseñanza, Nueva colección Labor, 1970.
} 


\subsection{Algunos aspectos que deben ser tenidos en cuenta al construir sistemas computarizados de ayuda a la docencia}

En el diseño y evaluación de sistemas de Educación Asistida por Computador deben tenerse en cuenta algunos aspectos:

- En un sistema de EAC pueden distinguirse claramente dos partes: el material didáctico y el software de base. De acuerdo con experiencias de grupos de trabajo sobre el tema, es importante que el primero de ellos sea desarrollado por el profesor de la materia correspondiente y el segundo por un experto en Informática. Los proyectos que no tengan en cuenta este principio básico tienen una gran probabilidad de fracasar.

- Para dar una idea del tipo de función que podría desempeñan la tecnología computacional en el proceso educativo, podemos mencionan algunas de las actividades que debe desarrollar un buen profesor $^{131}$.

- Transmitir información en forma clara y concisa.

- Formular preguntas que inciten a la investigación y requieran que el estudiante use conocimientos recién adquiridos de una manera tal que demuestre su entendimiento del tema.

- Interpretar las respuestas de los estudiantes, identificar los posibles errores en ellas, y darle las explicaciones del caso.

- Y, lo más importante, realizar todo lo anterior en el ámbito del salón de clase, o de un diálogo tutorial.

Los principios anteriores nos permiten identificar algunos de los usos que se le pueden dar al computador en el contexto educativo, clasificar los diferentes sistemas, y entender sus potencialidades y limitaciones.

- Cuando se habla de sistemas de EAC, no siempre se muestra la existencia de los diferentes niveles de trabajo posibles, por lo que se pueden sacar conclusiones apresuradas y hacer falsas generalizaciones. Una de las conclusiones a las que hemos llegado en nuestro grupo de trabajo es que entre más se baje en el grado de la enseñanza (de la universidad al bachillerato, la primaria y el preescolar) más ambiciosos son los proyectos (de diseñar sistemas de soporte para el curso de Álgebra Lineal en la universidad, a diseñar sistemas para enseñar a pensar a un preescolar hay una gran diferencia). Esto está de acuerdo con la observación de Piaget: "entre más jóvenes son los alumnos, más dificultades implica la enseñanza, si se la toma en serio" ${ }^{\prime 32}$.

Creemos que vale la pena experimentar en los diferentes niveles pero con una gran cautela y seriedad, y teniendo en cuenta la enorme dificultad que implica trabajar en los grados inferiores. En nuestro grupo de trabajo hemos dirigido inicialmente la mayor parte de los esfuerzos hacia la educación universitaria, por ser la más sencilla, y porque nuestra experiencia nos permite movernos en ella con mayor propiedad. Además

\footnotetext{
${ }^{131}$ TAIT K., The building of a computer-based teaching system, Computers and education, An internatonal Journal, Pergamon Press, Editado por P. Smith, Volumen 8, Número 1, 1984.

${ }^{132}$ PIAGET J. Ou va I'education, Bibliothèque Médiations, Donoël Gonthier, 1972.
} 
hemos puesto especial énfasis en que se puedan obtener de los proyectos, además de productos "concretos", conclusiones de orden general.

Conviene además tener en cuenta que la tecnología computacional puede aplicarse a otros sectores como la educación intermedia, el entrenamiento de personal, etc.

-Es importante no perder de vista el contexto general en que se van a aplicar los diferentes sistemas educativos (lo cual, entre otras cosas, es aplicable a cualquier proceso automatizado). A pesar de ser un principio muy conocido y evidente, vale la pena mencionarlo pues es fundamental-

\section{Conclusiones}

De las ideas expuestas en este artículo podemos sacar algunas conclusiones generales:

-Es importante que en el país se emprendan investigaciones y desarrollos sobre el tema de la Informática educativa con el fin de que podamos ir "apropiándonos" de las nuevas tecnologías, de que podamos sacar nuestras propias conclusiones sobre el asunto, y de evitar que se aumente la dependencia con respecto a los países desarrollados.

— Las posibilidades de aplicación de la tecnología computacional en el sector educativo son inmensas. Sin embargo, para que ésta pueda aportar soluciones eficaces y razonables en nuestro medio, es necesario que tengamos un buen conocimiento de sus posibilidades, de los problemas educativos y psicológicos, y de su contexto natural.

- Aunque no fueron tratados explícitamente en este artículo, hay una serie de problemas de orden práctico sin cuya solución es muy posible que fracase cualquier proyecto de investigación y desarrolle en el sector educativo (aunque no se utilice para nada el computador). Dentro de éstos podemos mencionar la escasez de recursos, la necesidad de preparar a los docentes y de infundirles una auténtica motivación en el asunto, la interferencia perniciosa de la política, etc.

SUMARIO: Este informe muestra la incidencia del conductismo y del estructuralismo (Skinner, Piaget, Papert), en el enfoque de la enseñanza asistida por computador. Presenta una síntesis de los aspectos que deben tenerse en cuenta cuando se hace diseño de instrucción con la anterior metodología y para algunos ejemplos se refiere a la experiencia llevada a cabo en el Departamento de Lenguas de la Universidad de Los Andes.

DESCRIPTORES: Informática, Enseñanza Programada, Enseñanza mediante computadora. Enseñanza de una Segunda Lengua, Educación Superior. 